\title{
Scrummi: Um processo de gestão ágil baseado no Scrum e aderente ao CMMI
}

\author{
Ana Sofia Cysneiros Marçal ${ }^{1}$, Maria Elizabeth Sucupira Furtado ${ }^{1}$ \\ ${ }^{1}$ Universidade de Fortaleza (UNIFOR) \\ Av. Washington Soares, 1321 - Fortaleza - Ceará - Brazil \\ anasofia.marcal@hotmail.com, elizabet@unifor.br
}

\begin{abstract}
Software development organizations that have been employing capability maturity models such as CMMI for improving their processes are now increasingly interested in the possibility of adopting agile development methods. This research embraced the challenge of analyzing the adherence of Scrum to CMMI practices for definition of an agile project management process, called Scrummi, built from an extension of the Scrum to be compliant with the CMMI project management process areas. The Scrummi was applied in a project of a CMMI level 3 company showing that agility and maturity can be applied together. Through Scrummi improvements were achieved related to increase in productivity, high commitment and development of project team.
\end{abstract}

Resumo. Organizações de software que têm empregado esforços na melhoria dos seus processos com base em modelos de qualidade tais como o CMMI, têm demonstrado um interesse crescente na adoção de métodos ágeis. Este trabalho adotou o desafio de analisar a aderência do Scrum em relação ao CMMI e definir o processo de gestão ágil Scrummi a partir de uma extensão do Scrum sendo aderente às áreas de gestão do CMMI. O Scrummi foi aplicado em um projeto de desenvolvimento de software em uma organização CMMI nível 3, mostrando que agilidade e maturidade podem caminhar juntas. A partir do Scrummi foram alcançadas melhorias relacionadas com aumento de produtividade, desenvolvimento e comprometimento do time do projeto.

\section{Introdução}

Ao longo dos últimos anos, organizações vêm adotando modelos de qualidade focados na maturidade do processo de software, tal como o CMMI (Capability Maturity Model Integration) [Chrissis et al. 2007]. Uma das razões para isso está relacionada ao fato de que a adequação e a aderência dos processos organizacionais aos níveis de maturidade destes modelos vêm garantindo melhorias relacionadas com o desempenho dos projetos bem como maior satisfação do cliente [Alleman 2004]. Por outro lado, os resultados, nem sempre positivos dos projetos de software, alinhados a uma necessidade de processos flexíveis, vêm motivando o estabelecimento de abordagens ágeis para o desenvolvimento de aplicações. De acordo com Boehm (2006), o surgimento de métodos ágeis no final dos anos 90 entre eles, o eXtreme Programming (XP) [Beck 1999] e o Scrum [Schwaber 2004], configurou uma tendência para o desenvolvimento ágil de aplicações a partir de 2000. Princípios ágeis surgem impulsionando o Gerenciamento Ágil de Projetos [Highsmith 2004] para auxiliar uma equipe de projeto a gerenciar e construir produtos inovadores e adaptáveis em um ambiente desafiador. 
Observando este cenário de mudanças, organizações que empregam um grande esforço na melhoria dos seus processos baseadas no CMMI começam a questionar por simplificação dos seus processos [Boehm e Turner 2004]. Com o intuito de se encontrar soluções para promover simplicidade no desenvolvimento de software em organizações que adotam modelos de maturidade, vários estudos e análises vêm sendo realizados desde o início dos anos 2000. Vários autores apresentam diferenças e semelhanças nas duas abordagens: desenvolvimento tradicional de software versus desenvolvimento ágil de software. Turner e Jain (2002) comentam que, apesar da existência de características distintas entre os métodos ágeis e o modelo CMMI, ambos buscam o melhor para que uma organização produza software com qualidade. Boehm e Turner (2004) relatam que a agilidade e disciplina são valores complementares no desenvolvimento $\mathrm{e}$ gerenciamento de software, e complementam dizendo que desenvolvedores disciplinados ou "plan-driven" devem ser ágeis. Da mesma forma, desenvolvedores ágeis devem ser disciplinados. Trabalhos mais recentemente publicados como em [Anderson 2005], [Dalton 2006], [Dutton 2006], [Davis et al. 2007] e [Glazer et al. 2008] apresentam análises detalhadas realizadas sobre o impacto do uso de metodologias ágeis na implementação de processos, considerando cada área de processo definida no CMMI. Estes trabalhos indicam não apenas ser viável a abordagem de se unir princípios ágeis ao CMMI, como também apontam esta abordagem híbrida como uma boa estratégia para alcance de melhores resultados em termos de qualidade e produtividade.

Seguindo esta tendência e acreditando-se na hipótese de que é possível combinar agilidade com modelos de maturidade, este trabalho adotou o desafio de analisar a aderência do método ágil Scrum em relação ao CMMI, especificamente no que diz respeito às práticas específicas dos processos de gerenciamento de projetos. Esta análise foi seguida por uma investigação do real interesse de organizações de software em incorporar na gestão de seus projetos práticas de métodos ágeis e CMMI. Após uma breve explicação sobre o CMMI e o Scrum descrita na seção 2 deste artigo, a seção 3 apresenta os resultados desta investigação que serviram como motivação para se definir um processo de gestão ágil de projetos, o qual combina práticas do Scrum com práticas do CMMI. Tal processo, chamado Scrummi, é apresentado na seção 4. A aplicação do Scrummi, descrita na seção 5, permite comprovar a possibilidade de adoção de práticas ágeis dentro de um contexto de maturidade contribuindo para a melhoria dos processos organizacionais e aumento de produtividade. Por fim, as principais contribuições e trabalhos futuros são descritos na seção 6 .

\section{Enfoques sobre CMMI e Agilidade}

$\mathrm{Na}$ área de desenvolvimento de software e tecnologia da informação, várias metodologias, modelos e processos de software trazem métodos, técnicas, práticas, ferramentas e atividades relacionadas com gerência de projetos. Entre eles, destaca-se o CMMI [Chrissis et al. 2007] o qual possui uma categoria específica para o Gerenciamento de Projetos englobando atividades de gestão relacionadas com planejamento, monitoramento e controle do projeto.

Para descrever as interações entre as áreas de processo de gestão do CMMI é mais útil tratá-las em dois grupos: gerenciamento de projetos fundamentais e gerenciamento de projetos avançados como descritos em [Chrissis et al. 2007]. As áreas de processo de gerenciamento de projetos fundamentais (compostas pelas áreas de 
processo Planejamento de Projeto, Controle e Monitoramento de Projeto e Gerenciamento de Acordos com Fornecedores) endereçam as atividades relacionadas ao estabelecimento e manutenção do plano do projeto, estabelecimento e manutenção de compromissos, monitoramento de progresso do projeto em relação ao planejado, implementação de ações corretivas e gerenciamento de acordos com os fornecedores.

As demais áreas de processo relativas ao gerenciamento de projetos do CMMI (Gerenciamento Integrado de Projetos + Desenvolvimento Integrado do Produto e do Processo, Gerenciamento de Riscos e Gerenciamento de Projetos Quantitativo) compõem o gerenciamento de projetos avançados. Estas áreas de processos endereçam atividades para estabelecer um processo definido para projetos em desenvolvimento, estabelecer um ambiente de trabalho a partir de padrões do ambiente organizacional, coordenar e colaborar com os stakeholders relevantes, gerenciar riscos, formar e sustentar times integrados na condução de projetos e gerenciar quantitativamente o processo definido para os projetos sendo conduzidos.

Os métodos ágeis surgiram como uma reação aos modelos clássicos e tradicionais de desenvolvimento, destacando a necessidade de realizar documentações completas [Beck et al. 2001]. O Scrum foi o método ágil escolhido neste trabalho, porque sua maior ênfase é dada ao gerenciamento de um projeto e reúne atividades de monitoramento e feedback, em geral, visando a identificação e correção de quaisquer impedimentos num processo de desenvolvimento.

O Scrum define um framework iterativo e incremental cujas atividades são assumidas por três papéis principais: Product Owner, ScrumMaster e Time. Um projeto no Scrum se inicia com uma visão do produto que será desenvolvido. Em seguida, o Product Backlog é criado contendo a lista de todos os requisitos conhecidos. O Product Backlog é então priorizado e dividido em releases. No Scrum todo o trabalho é realizado em iterações de duração fixa, chamadas de sprints. Schwaber (2004) explica que cada sprint se inicia com uma reunião de planejamento na qual o Product Owner e o time decidem em conjunto o que deverá ser implementado. A reunião é dividida em duas partes. Na primeira parte, o Product Owner apresenta os requisitos de maior valor e prioriza aqueles que devem ser implementados. $O$ time então define colaborativamente o que poderá entrar no desenvolvimento da próxima sprint, considerando sua capacidade de produção. Na segunda parte, o time planeja seu trabalho, definindo o Sprint Backlog, que são as tarefas necessárias para implementar as funcionalidades selecionadas a partir do Product Backlog. Todo trabalho realizado numa sprint é devidamente acompanhado e inspecionado em reunião de revisão e em reunião de retrospectiva, com o objetivo de melhorar o processo, o time e/ou o produto.

\subsection{Combinando Agilidade e CMMI}

Existem várias iniciativas e trabalhos publicados relacionados com a melhoria dos processos de desenvolvimento de software baseados em metodologias ágeis e CMMI. Muitos deles focam em responder questionamentos em torno da compatibilidade do desenvolvimento de software ágil e do CMMI, bem como da possibilidade de ser ao mesmo tempo ágil e disciplinado [Paulk 2001], [Orr 2002], [Turner e Jain 2002], [Boehm e Turner 2004], [Dutton 2006], [Dalton 2006] e [Glazer et al. 2008]. 
Em [Vriens 2003] uma experiência é apresentada sobre uma empresa que desejava alcançar o nível 2 de maturidade do SW-CMM e a certificação ISO9001, considerando o uso combinado do XP e Scrum. O método desenvolvido pela empresa foi chamado Xp@Scrum e os métodos ágeis foram combinados da seguinte forma: XP foi usado para os processos técnicos de engenharia de software e após 1 ano o Scrum foi introduzido para apoiar questões gerenciais. $O$ sucesso alcançado foi total, pois a companhia conseguiu comprovar a aderência a ambos os modelos de qualidade.

Zannata (2004) apresenta uma proposta de extensão do método ágil Scrum, o xScrum, para a gerência e desenvolvimento de requisitos visando adequação ao CMMI. Zannata inicia seu trabalho fazendo uma avaliação do método ágil Scrum segundo as perspectivas das áreas de processo do CMMI relacionadas com gestão de requisitos. Em seguida propõe o xScrum e sua aplicação em um ambiente de desenvolvimento de software real.

Outro trabalho bastante interessante e prático relacionado com a compatibilidade entre agilidade e CMMI foi publicado pela Microsoft em 2005 e relata a experiência da empresa na adequação do seu método para desenvolvimento ágil de software, o MSF, por meio da adoção dos ensinamentos de W. Edwards Demming, para ficar aderente ao nível 3 de maturidade do CMMI. Segundo Anderson (2005), o resultado deste trabalho compreendeu a definição de um método de planejamento adaptável, altamente iterativo e com pouca documentação e fortemente automatizado por meio de ferramentas.

Davis e seus colegas [Davis et al. 2007] descrevem em seu trabalho o Agile +, uma abordagem híbrida para o desenvolvimento de software baseada nos elementos centrais do XP e aderente ao CMMI nível 3. O Agile+ parte do XP e faz uma extensão para incluir algumas das melhores práticas tradicionais de engenharia de software preservando a essência da agilidade.

Em [Sutherland et al. 2007] apresenta-se como uma organização CMMI nível 5 usou o Lean Software Development [Poppendieck 2006], como elemento direcionador para a otimização do seu programa de melhoria contínua. Ressalta experiências valiosas foram adquiridas ao combinar práticas do Scrum e XP com o CMMI nível 5, mostrando que os projetos que usam esta abordagem híbrida são mais bem sucedidos na melhoria da qualidade de software e atendem às necessidades dos clientes de forma mais rápida e eficaz. Entre os benefícios da abordagem adotada cita que a produtividade em equipes do Scrum é quase duas vezes superior a de equipes tradicionais e que uma abordagem de testes aplicada em alguns projetos baseada em estórias reduziu os defeitos encontrados na fase final de testes em $38 \%$.

Com relação ao gerenciamento de projetos, Leal (2008) propõe uma abordagem ágil para o gerenciamento de projetos de software baseada no PMBOK [PMI 2004]. O Agilus é um modelo híbrido para o gerenciamento de projetos que mescla a gestão clássica com a ágil em prol do gerenciamento e desenvolvimento de projetos de sucesso, na visão do cliente e do time do projeto.

Até a realização desta dissertação, o mais recente trabalho relacionado com a combinação de métodos ágeis e CMMI foi publicado pelo SEI em 2008. O relatório técnico divulgado esclarece razões pelas quais contradições e discórdias entre práticas ágeis e CMMI não precisam existir e propõe a união das duas abordagens a fim de se obter uma melhoria do desempenho empresarial [Glazer et al. 2008]. Os autores do 
relatório destacam que o CMMI e agilidade podem ser usados conjuntamente e com sucesso e referencia várias experiências do uso das duas abordagens em conjunto.

Observa-se, entretanto, que nenhum destes trabalhos define um processo genérico para a gestão de projetos, baseado no Scrum, alinhado com os princípios, valores e práticas do Gerenciamento Ágil de Projetos e ao mesmo tempo compatível com o CMMI. Acredita-se que a definição deste processo é relevante tanto para empresas que possuem seus processos baseados no modelo CMMI e estão planejando a melhoria dos seus processos de gestão por meio da introdução de agilidade, bem como para empresas que estão pensando em definir um novo processo de gestão de projetos baseado na combinação de práticas do CMMI e Scrum.

\section{Investigações sobre Aderência e Combinação do Scrum e CMMI}

Esta seção descreve os caminhos que nortearam a pesquisa ao investigar as seguintes questões: Que práticas das áreas de processo de Gestão de Projetos do CMMI estão alinhadas às práticas do Scrum? e Qual o interesse de organizações brasileiras de desenvolvimento de software pela adoção de processos híbridos? Os resultados das investigações resultaram em publicações em conferências e periódicos [Marçal et al. 2007a], [Marçal et al. 2008a] e [Marçal et al. 2008b] como descritos resumidamente a seguir.

A análise de aderência entre as práticas do Scrum e práticas do CMMI considerou as áreas de processo do modelo de Gestão de Projetos: Planejamento do Projeto (PP), Controle e Monitoramento do Projeto (PMC), Gerenciamento de Acordos com Fornecedores (SAM), Gerenciamento Integrado de Projetos (IPM), Gerenciamento de Riscos (RSKM) e Gerenciamento Quantitativo do Projeto (QPM). Para cada uma das práticas das áreas de processo foi realizada uma classificação segundo os critérios de satisfação: Não Satisfeita (Não há evidências da prática no Scrum), Parcialmente Satisfeita (Há evidências da prática no Scrum, embora a prática não esteja plenamente atendida) e Satisfeita (A prática está totalmente atendida no Scrum). Após a fase de classificação, foi calculado o percentual de satisfação de cada área de processo, tomando como base o número total de práticas específicas da área de processo em análise. Em seguida foi gerada uma visão consolidada da cobertura do Scrum nas áreas de processo de gestão de projetos do CMMI, conforme visualizados na Figura 1.

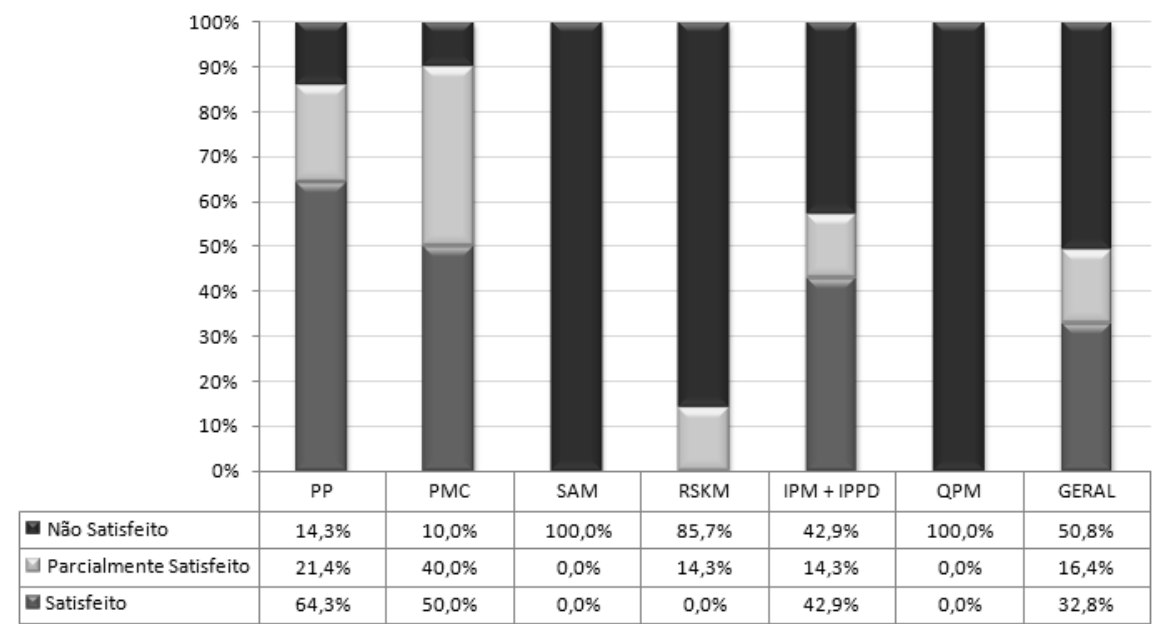

Figura 1: Cobertura do Scrum nas PAs de Gerenciamento de Projetos do CMMI [Marçal et al. 2007a] 
Uma proposta preliminar de extensão do Scrum foi definida em [Marçal et al. 2007b] priorizando inicialmente a resolução das principais divergências do Scrum com relação às práticas de estimativas, riscos e gerenciamento de problemas e ações corretivas do modelo CMMI. Foi neste momento que se investigou como as empresas brasileiras gerenciavam tais lacunas. $\mathrm{O}$ instrumento usado para esta investigação foi uma pesquisa aplicada por meio de um formulário publicado na web contendo informações sobre: a empresa; o processo de desenvolvimento adotado na empresa; o perfil de projetos que já usaram Scrum; e como era realizada a melhoria dos seus processos. Participaram da pesquisa 73 empresas localizadas em todas as regiões do Brasil. Das empresas pesquisadas, $44 \%$ possuem até 10 anos de mercado, $37 \%$ possuem entre 11 e 40 anos e apenas $19 \%$ possuem acima de 40 anos de mercado. Com relação ao tamanho da organização, em número aproximado de profissionais envolvidos com a gestão e o desenvolvimento de projetos, $18 \%$ das empresas pesquisadas possuem até 9 profissionais, $30 \%$ possuem entre 10 a 49 pessoas, $8 \%$ possuem entre 50 a 99 pessoas e $44 \%$ possuem acima de 100 pessoas.

Analisando-se os resultados da pesquisa percebe-se que a adoção de práticas ágeis em processos de desenvolvimento de software é uma tendência tanto em empresas que possuem processos aderentes ao CMMI quanto em empresas que desejam alcançar algum nível de maturidade do modelo. Tal fato aponta uma demanda real para a definição de uma solução híbrida para o gerenciamento de projetos que promova flexibilidade e agilidade combinada à disciplina necessária para alcançar os níveis de maturidade do CMMI.

\section{Scrummi: Um processo de gestão baseado no Scrum e aderente ao CMMI}

O Scrummi [Marçal 2008c] é um processo de gestão ágil de projetos sendo baseado em extensões do método ágil Scrum a partir das seguintes áreas de processo de gerenciamento de projeto do CMMI: PP, PMC, IPM e RSKM. Foram excluídas do escopo do Scrummi, SAM e QPM, já que estas áreas de processo nem sempre são aplicadas a todas as organizações e têm uma importância menor dentro do contexto de gestão de projetos ágeis. O Scrummi possui uma apresentação publicada em formato HTML de acordo com os padrões do EPF Composer e está disponível para ser acessado no site http://www.scrummi.com.br.

O Scrummi foi desenvolvido com o propósito de incorporar soluções simples para as divergências e lacunas encontradas na investigação da cobertura do Scrum versus o CMMI [Marçal 2007a], dentre as quais se destacam:

- Ausência de técnicas ou práticas alternativas para a realização das estimativas do projeto, solucionada com a introdução de atividades para estimar complexidade em Story Points suportada por artefatos e guias;

- Lacunas no planejamento e gerenciamento do orçamento do projeto, em que a solução está relacionada com atividades no processo para estimar e monitorar a duração, esforço e custos do projeto de forma simplificada;

- Ausência de um melhor gerenciamento dos riscos para o qual foram introduzidas atividades e guias específicos relacionados com preparação, identificação e análise de riscos e suas ações de mitigação, apoiada por um template para a lista de riscos com as mínimas informações necessárias ao gerenciamento dos riscos; 
- Lacunas no gerenciamento de ações corretivas de problemas e dependências supridas por meio da complementação da lista de impedimentos Scrum, com adição de informações necessárias para fazer o gerenciamento de ações corretivas até o seu fechamento;

- Ausência de um planejamento e monitoramento dos dados do projeto, solucionada com a extensão do Product Backlog, transformando-o no Backlog do Projeto que, além de requisitos funcionais, contemplará requisitos ambientais do projeto, relacionados com a gestão dos dados, infra-estrutura e capacitações;

- Lacunas no gerenciamento integrado do projeto devido à ausência de um processo integrado e definido que é adaptado a partir do conjunto de processos padrão da organização, preenchida pelo estabelecimento da abordagem de execução do projeto o qual inclui a definição de um processo adaptado para o projeto baseado no processo organizacional, bem como a criação de artefato simples para a base histórica de projetos que deverá ser atualizado e consultado ao longo da execução do projeto.

\subsection{Papéis, Artefatos e Atividades do Scrummi}

O Scrummi define, além dos três papéis já definidos no Scrum, o papel do Gerente Sênior de Projetos, o qual é responsável por prover os recursos organizacionais necessários para a execução dos projetos, bem como realizar o acompanhamento do progresso do projeto. O papel do Gerente de Projetos no Scrummi, corresponde a uma extensão do papel do ScrumMaster, acumulando responsabilidades adicionais de gestão de riscos e de custos do projeto.

No Scrummi foram definidos nove artefatos os quais representam a documentação mínima e necessária para a gestão ágil do projeto aderente às práticas do CMMI. O Quadro 1 apresenta os principais artefatos e uma breve descrição do seu conteúdo. Além desses, existem templates para: base histórica de projetos e atas de reunião de planejamento, revisão e retrospectiva.

\section{Quadro 1: Artefatos do Scrummi}

\begin{tabular}{|l|l|}
\hline Artefato & Conteúdo \\
\hline $\begin{array}{l}\text { Plano do } \\
\text { Projeto }\end{array}$ & $\begin{array}{l}\text { Contém o planejamento macro do projeto: Visão Geral do Projeto; Comunidade do } \\
\text { Projeto; Plano de Comunicação e Colaboração do Projeto; Abordagem de Execução } \\
\text { do Projeto. }\end{array}$ \\
\hline $\begin{array}{l}\text { Backlog do } \\
\text { Projeto }\end{array}$ & $\begin{array}{l}\text { Contém além dos requisitos do produto (funcionais e não funcionais), solicitações de } \\
\text { mudanças. Adicionalmente o Backlog do Projeto dispõe de informações para } \\
\text { monitorar o projeto, acompanhar as estimativas e custos do projeto além de definir e } \\
\text { acompanhar o plano de entreqas. }\end{array}$ \\
\hline $\begin{array}{l}\text { Backlog da } \\
\text { Sprint }\end{array}$ & $\begin{array}{l}\text { Informações necessárias para o planejamento e monitoramento da sprint, incluindo } \\
\text { lista de atividades que devem ser realizadas pelo time do projeto e o gráfico de } \\
\text { consumo da sprint. }\end{array}$ \\
\hline Lista de Riscos & $\begin{array}{l}\text { Informações necessárias para a identificação, análise e gerenciamento dos riscos e } \\
\text { suas ações de mitigação. }\end{array}$ \\
\hline Impedimentos & $\begin{array}{l}\text { Informações necessárias para gerenciamento dos problemas e dependências do } \\
\text { projeto e de suas ações corretivas garantindo acompanhamento até o seu } \\
\text { fechamento. }\end{array}$ \\
\hline
\end{tabular}

As atividades do Scrummi foram organizadas segundo as cinco fases do framework do Gerenciamento Ágil de Projetos como ilustra a Figura 2. 
A fase Visão do Scrummi representa o primeiro nível do planejamento e tem como objetivo principal determinar a visão geral projeto que estará sendo desenvolvido (isto inclui a visão do produto, comunidade do projeto e seu plano de comunicação, bem como a abordagem de execução do projeto) para em seguida, definir um backlog inicial para o projeto. Após a fase Visão, são executadas as fases Especulação, Exploração e Adaptação para cada sprint, com o objetivo de refinar o produto do projeto. $\mathrm{Na}$ fase Especulação é realizado o segundo nível do planejamento do projeto, o qual inclui a atualização e estimativa do Backlog do Projeto, bem como planejamento das entregas e marcos do projeto e de cada sprint. O planejamento de uma sprint tem por objetivo estabelecer o terceiro nível do planejamento do projeto, sendo composto pelas atividades: definir objetivo e escopo da sprint (Sprint Planning 1) e construir backlog da sprint (Sprint Planning 2). A fase Exploração compreende o desenvolvimento e entrega de requisitos prontos de maior valor agregado ao cliente em um intervalo de tempo fixo, assim como o monitoramento constante dos riscos visando reduzir a incerteza do projeto. Nesta fase são realizadas atividades para monitorar a sprint e promover o desenvolvimento do time, explorando o enfoque ágil de gestão baseado na colaboração e criando-se um espaço para a liderança participativa. A fase Adaptação, realizada ao final da sprint, engloba a revisão dos resultados entregues, a análise da situação atual e do desempenho do time do projeto para eventual adaptação do processo e/ou requisitos do sistema/produto. Uma vez obtido o produto final, a fase Encerramento é realizada, com a transferência dos aprendizados-chave e o aceite final do projeto para a sua conclusão.

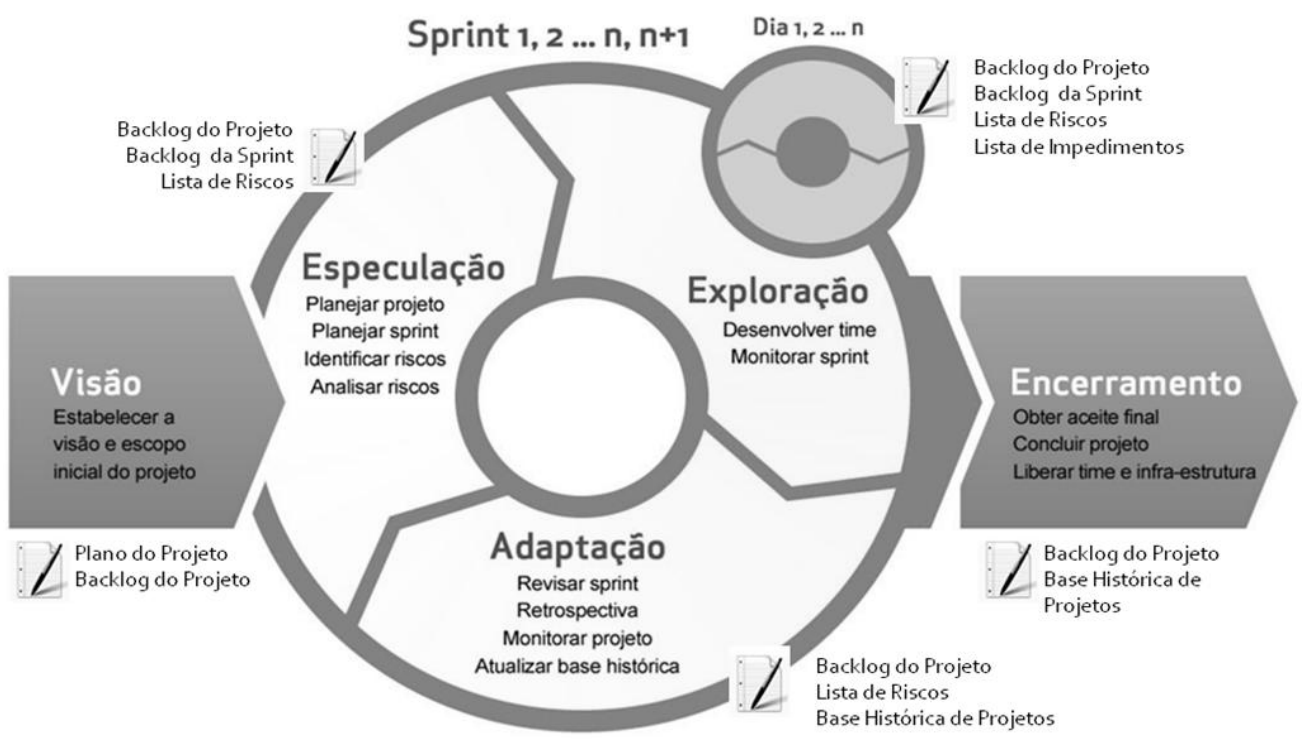

Figura 2: Framework de atividades do Scrummi [Marçal 2009b]

\subsection{Considerações sobre a Aderência do Scrummi ao CMMI}

Com relação às lacunas existentes e que impactam diretamente no planejamento e monitoramento do projeto representado pelas áreas de processo PP e PMC, o Scrummi conseguiu resolvê-las de forma que todas as práticas podem agora ser classificadas como Satisfeitas, como mostra o Quadro 2. A classificação foi realizada usando-se os critérios de satisfação definidos em [Marçal et al. 2007a]. O mesmo acontece com todas as lacunas relacionadas com o gerenciamento de riscos e que afetam diretamente as 
práticas de RSKM, já que atividades específicas apoiadas por guias e templates foram definidas no processo visando identificar e analisar os riscos do projeto bem como definir e acompanhar suas ações de mitigação e contingência.

\section{Quadro 2: Classificação das práticas do CMMI x Scrummi}

\begin{tabular}{|c|c|c|c|}
\hline \multirow{4}{*}{$\begin{array}{l}\text { Principais lacunas } \\
\text { Ausência de técnicas } \\
\text { ou práticas alternativas } \\
\text { para a realização das } \\
\text { estimativas do projeto }\end{array}$} & \multicolumn{2}{|c|}{ Práticas CMMI impactadas } & \multirow{2}{*}{\begin{tabular}{|l|} 
Classificação \\
Satisfeita
\end{tabular}} \\
\hline & \multirow[t]{2}{*}{ PP } & $\begin{array}{l}\text { SP } 1.2 \text { Estabelecer Estimativas de Atributos de Produtos de } \\
\text { Trabalho e Tarefa }\end{array}$ & \\
\hline & & SP 1.4 Determinar Estimativas de Esforço e Custo & Satisfeita \\
\hline & PMC & SP 1.1 Monitorar Parâmetros de Planejamento do Projeto & Satisfeita \\
\hline \multirow{3}{*}{$\begin{array}{lr}\text { Lacunas no } \\
\text { planejamento } \\
\text { gerenciamento do } \\
\text { orçamento do projeto }\end{array}$} & \multirow[t]{2}{*}{ PP } & SP 1.4 Determinar Estimativas de Esforço e Custo & Satisfeita \\
\hline & & SP 2.1 Estabelecer Orçamento e Cronograma & Satisfeita \\
\hline & PMC & SP 1.1 Monitorar Parâmetros de Planejamento do Projeto & Satisfeita \\
\hline \multirow{9}{*}{$\begin{array}{l}\text { Ausência de um } \\
\text { melhor gerenciamento } \\
\text { dos riscos }\end{array}$} & PP & SP 2.2 Identificar Riscos do Projeto & Satisfeita \\
\hline & PMC & SP 1.3 Monitorar os Riscos do projeto & Satisfeita \\
\hline & \multirow[t]{7}{*}{ RSKM } & SP 1.1 Determinar Fontes e Categorias do risco & Satisfeita \\
\hline & & SP 1.2 Determinar os parâmetros do risco & Satisfeita \\
\hline & & SP 1.3 Estabelecer estratégia de gerenciamento dos riscos & Satisfeita \\
\hline & & SP 2.1 Identificar Riscos & Satisfeita \\
\hline & & SP 2.2 Avaliar, categorizar e priorizar riscos & Satisfeita \\
\hline & & SP 3.1 Desenvolver planos de mitigação de riscos & Satisfeita \\
\hline & & SP 3.2 Implementar planos de mitigação de riscos & Satisfeita \\
\hline \multirow{4}{*}{$\begin{array}{lr}\text { Lacunas } & \text { no } \\
\text { gerenciamento } & \text { de } \\
\text { ações corretivas } & \text { de } \\
\text { problemas } & \text { e } \\
\text { dependências } & \end{array}$} & \multirow[t]{2}{*}{ PMC } & SP 2.2 Tomar ações corretivas & Satisfeita \\
\hline & & SP 2.3 Gerenciar ações corretivas & Satisfeita \\
\hline & \multirow[t]{2}{*}{ IPM } & SP 2.2 Gerenciar Dependências & Satisfeita \\
\hline & & SP 2.3 Resolver Questões de Coordenação & Satisfeita \\
\hline \multirow{2}{*}{$\begin{array}{lr}\text { Ausência de } & \text { um } \\
\text { planejamento } & \mathrm{e} \\
\text { monitoramento } & \text { dos } \\
\text { dados do projeto } & \end{array}$} & PP & SP 2.3 Planejar o Gerenciamento de Dados & Satisfeita \\
\hline & PMC & SP 1.4 Monitorar o gerenciamento dos Dados & Satisfeita \\
\hline \multirow{6}{*}{$\begin{array}{l}\text { Lacunas no } \\
\text { gerenciamento } \\
\text { integrado do projeto } \\
\text { devido à ausência de } \\
\text { um processo integrado } \\
\text { e definido que é } \\
\text { adaptado a partir do } \\
\text { conjunto de processos } \\
\text { padrão da organização }\end{array}$} & \multirow[t]{6}{*}{ IPM } & SP 1.1 Estabelecer o Processo Definido do Projeto & $\begin{array}{l}\text { Parcialmente } \\
\text { Satisfeita }\end{array}$ \\
\hline & & $\begin{array}{l}\text { SP 1.2 Utilizar os Ativos de Processos Organizacionais } \\
\text { para o planejamento das atividades do projeto }\end{array}$ & $\begin{array}{l}\text { Parcialmente } \\
\text { Satisfeita }\end{array}$ \\
\hline & & SP 1.3 Estabelecer o Ambiente de trabalho do projeto & $\begin{array}{l}\text { Parcialmente } \\
\text { Satisfeita }\end{array}$ \\
\hline & & SP 1.4 Integrar os Planos & $\begin{array}{l}\text { Parcialmente } \\
\text { Satisfeita }\end{array}$ \\
\hline & & SP 1.5 Gerenciar o Projeto Utilizando os planos Integrados & $\begin{array}{l}\text { Parcialmente } \\
\text { Satisfeita }\end{array}$ \\
\hline & & SP 1.6 Contribuir para Ativos de Processos Organizacionais & $\begin{array}{l}\text { Parcialmente } \\
\text { Satisfeita }\end{array}$ \\
\hline
\end{tabular}

Entretanto, apesar de o Scrummi ter inserido no seu processo atividades genéricas e bastante simplificadas para estabelecer a abordagem de execução do processo (incluindo a definição do processo do projeto) e de ter definido um artefato 
simples para a base histórica de projetos, o Scrummi não é auto-suficiente para atender todas as práticas de IPM. Especialmente as que afetam a primeira meta relacionada com o estabelecimento e gerenciamento de um projeto de acordo com um processo organizacional (definido e mais abrangente que inclua todas as disciplinas e atividades necessárias para adquirir, desenvolver ou manter o produto), o qual é adaptado a partir do conjunto de processos padrão da organização em questão. Esta decisão foi proposital. Acredita-se que a definição de um processo organizacional completo deve ser executada considerando-se a realidade de cada empresa, estando esse processo alinhado às estratégias, maturidade e capacidades da organização, o que o torna bem específico. Sendo assim, sugere-se que as atividades do Scrummi sejam complementadas com as definições, guias e critérios de adaptações dos processos organizacionais específicos das empresas.

\section{Aplicação do Scrummi}

A aplicação prática do Scrummi foi realizada em um projeto real de desenvolvimento de software numa instituição de pesquisa e desenvolvimento contando com um processo de desenvolvimento de software aderente ao CMMI nível 3. Para tanto iniciou um projeto DMADV (Define, Measure, Analyse, Design and Verify) tendo como principal objetivo melhorar a produtividade e simplificar os processos dos projetos de software por meio da introdução de práticas ágeis. Neste contexto, o estudo de caso realizado teve como objetivos principais: contribuir de forma relevante em organizações que têm um processo baseado no CMMI e estão planejando a sua melhoria e promover o aumento da produtividade dos times de desenvolvimento.

O projeto selecionado para o estudo de caso foi um projeto de desenvolvimento de um sistema de Gestão de Suprimentos para um cliente da indústria têxtil. O projeto deveria ser executado segundo a modalidade de fábrica de software, com escopo variável compreendendo um esforço de dez mil horas de trabalho consumidas em um banco de quinhentos Use Case Points. A autora deste trabalho assumiu o papel do Gerente do Projeto e contava com um time de desenvolvimento de tamanho médio, com aproximadamente doze pessoas incluindo todos os perfis necessários para a execução do projeto.

O projeto foi iniciado em agosto de 2008 e concluído em fevereiro de 2009 com duração final de sete meses. Todo o projeto foi realizado seguindo o ciclo de vida incremental e iterativo com sprints de 4 semanas como mostra a Figura 3. Ao todo foram executadas 8 sprints, incluindo a Sprint 0 (Fase Visão), na qual foi estabelecida a Visão Geral do Projeto com base nas informações e premissas definidas no contrato.

A estimativa de duração, esforço e custos do projeto oriundas do contrato foram refinadas considerando as restrições de prazo e custos do projeto. Os planejamentos das entregas e marcos do projeto foram realizados considerando-se a restrição de que o escopo dos requisitos era variável. Desta forma um planejamento preliminar bem simples foi estabelecido, com datas para a conclusão das sprints e escopo sendo definido ao longo do projeto. As datas de início e término das sprints foram definidas por meio da construção de um cronograma macro, o qual incluía os feriados e considerava 20 dias úteis para a execução de uma sprint, garantindo assim uma uniformidade de 4 semanas para a duração das sprints do projeto piloto em desenvolvimento. 


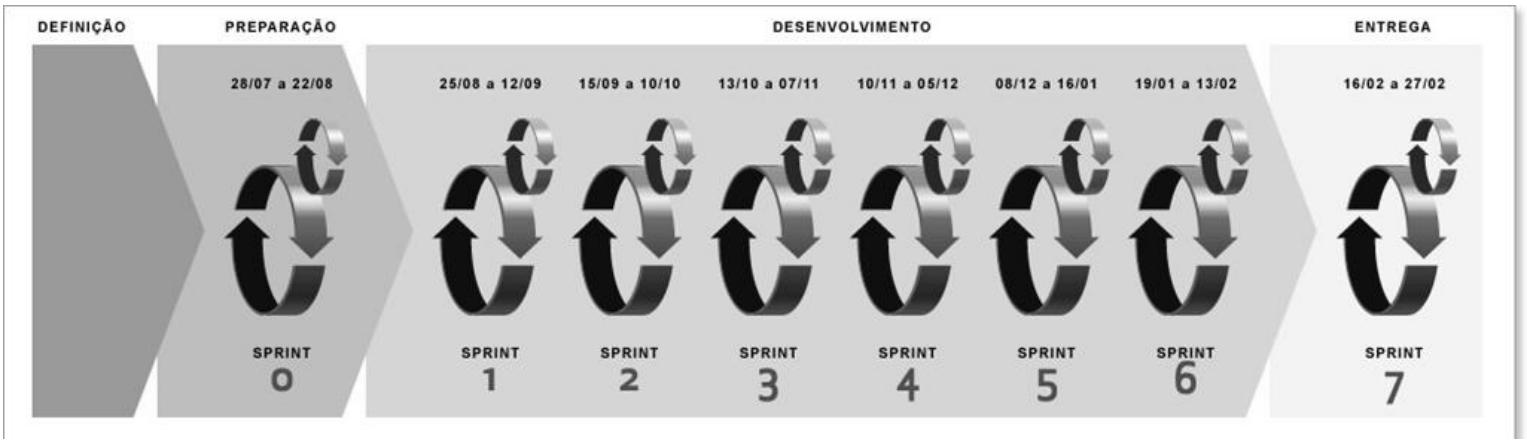

Figura 3: Macro Cronograma do Projeto

A alocação do time e estabelecimento da comunidade do projeto, bem como suas interfaces e plano de comunicação entre os participantes do projeto também foram realizados ao longo da sprint 0 . O processo definido para o projeto piloto foi adaptado a partir dos processos padrões da organização, considerando as atividades e artefatos do Scrummi, de forma que posteriormente, estas adaptações fossem incorporadas ao processo organizacional criando-se alternativas para se executar a gestão de projetos: ágil ou clássica.

A partir da sprint 1, a cada sprint eram realizadas atividades das fases Especulação, Exploração e Adaptação do Scrummi, como ilustradas na Figura 4.

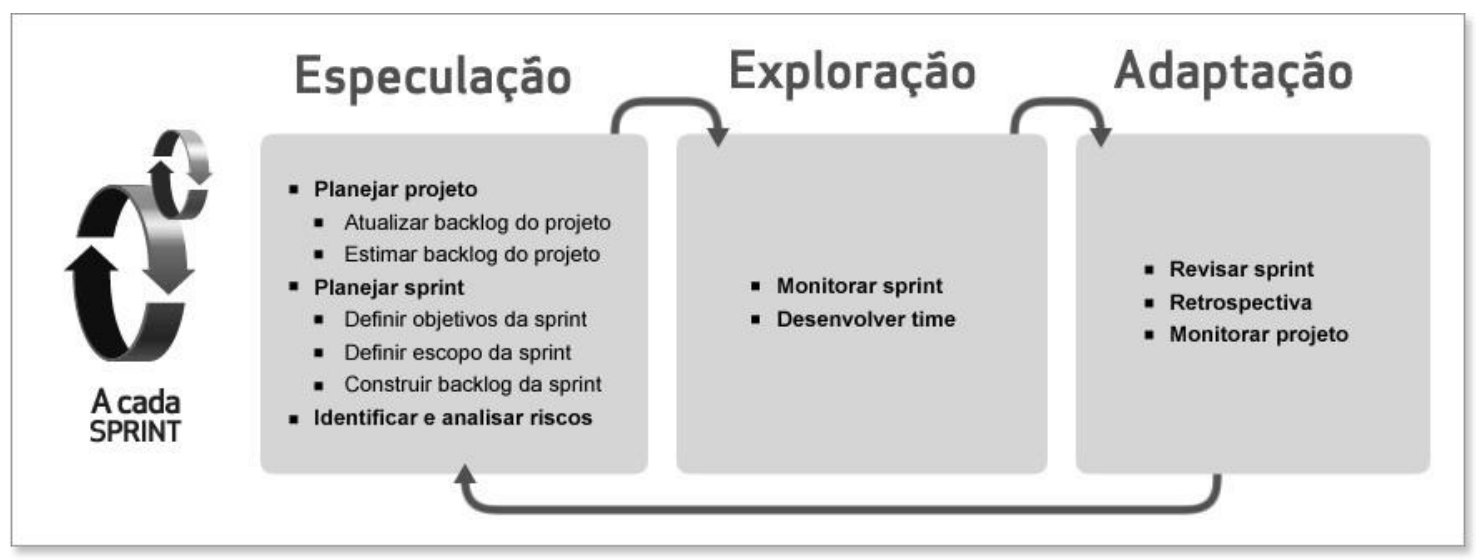

Figura 4: Atividades das fases Especulação, Exploração e Adaptação executadas no projeto piloto

Antes de iniciar uma sprint, o Backlog do Projeto era atualizado com novos requisitos funcionais (casos de uso) identificados para o projeto, bem como solicitações de mudanças, requisitos não funcionais ou requisitos ambientais (capacitações e necessidades de infra-estutura). As estimativas dos itens de Backlog do Projeto eram realizadas em Story Points e convertidas em Use Case Points, como descritas em [Marçal 2009a]. A definição do escopo da sprint realizada pelo time do projeto era revisado após a construção do Backlog da Sprint. Ajustes poderiam acontecer considerando as estimativas de esforço das atividades.

A definição do Backlog da Sprint era realizada durante a Reunião de Planejamento da Sprint - parte 2 na qual o time em conjunto com o Gerente do Projeto definia todo o trabalho (conjunto de atividades) necessário para a implementação do escopo da sprint, incluindo: atividades derivadas a partir do processo de desenvolvimento definido para o projeto e atividades adicionais complementares às 
atividades derivadas do processo (gestão do projeto, gestão de configuração, gestão de dados e gestão de riscos, capacitações e treinamentos, configuração e estabelecimento do ambiente de desenvolvimento).

As estimativas de esforço das atividades de processo eram derivadas a partir da complexidade dos casos de uso em Story Points, fazendo-se os ajustes necessários de acordo com dados históricos da sprint passada. A Figura 5 ilustra a planilha de estimativa usada na Sprint 4 do projeto. As estimativas (esforço) das demais atividades eram realizadas em conjunto com o time, levando-se em consideração os dados históricos de sprints passadas bem como opiniões de especialistas. Ao concluir a definição do Backlog da Sprint, suas atividades eram cadastradas e disponibilizadas na ferramenta organizacional JIRA, uma ferramenta web para monitoramento de bugs e de problemas. A ferramenta JIRA foi configurada e adaptada para realizar o cadastro e monitoramento das atividades do Backlog da Sprint, assim como o cadastro e acompanhamento dos impedimentos segundo os artefatos do Scrummi.

\begin{tabular}{|c|c|c|c|c|c|c|c|c|c|c|}
\hline \multirow[b]{3}{*}{ Atividades } & \multicolumn{10}{|c|}{ Estimativas em Horas } \\
\hline & \multicolumn{9}{|c|}{ Story Points } & \multirow[t]{2}{*}{ Fator de ajuste } \\
\hline & 1 & 2 & 3 & 5 & 8 & 13 & 21 & 34 & 55 & \\
\hline Análise e Projeto + Mapeamento OR + Geração Script Banco & 2,50 & 2,50 & 2,50 & 4,50 & 6,00 & 6,00 & 8,00 & 24,00 & 32,00 & 1 \\
\hline Revisão técnica A\&P e Correção de problemas - feita em pares & 0,00 & 1,00 & 1,00 & 1,00 & 2,00 & 2,00 & 2,00 & 3,00 & 4,00 & 1 \\
\hline Codificação Geração + Wizard + Codificação (RN + Interfaces) + Testes & & & & & & & & & & 1 \\
\hline Unitários + pré-teste sistêmico & 2,00 & 3,50 & 5,00 & 9,00 & 12,00 & 24,00 & 36,00 & 52,00 & 72,00 & \\
\hline Revisão técnica de código (find bugs + inspeção manual + correção & & & & & & & & & & 1,5 \\
\hline de problemas) & 1,50 & 1,50 & 1,50 & 1,50 & 1,50 & 3,00 & 3,00 & 4,50 & 4,00 & \\
\hline Especificação de testes & 2,50 & 2,50 & 4,00 & 6,00 & 6,00 & 10,00 & 13,00 & 13,00 & 16,00 & 1 \\
\hline Execução de testes sistêmicos & 3,00 & 3,00 & 3,00 & 6,00 & 6,00 & 8,00 & 10,00 & 10,00 & 12,00 & 1 \\
\hline Total de horas geral & $|11,50|$ & 14,00 & 17,00 & 28,00 & 33,50 & 53,00 & 72,00 & 106,50 & 140,00 & \\
\hline
\end{tabular}

\begin{tabular}{|c|c|c|c|c|c|c|c|c|c|c|}
\hline \multirow[t]{2}{*}{ Estimativa da Sprint 4} & \multicolumn{9}{|c|}{$S P$} & \multirow{2}{*}{$\begin{array}{r}\text { TOTAL } \\
\text { Planejado }\end{array}$} \\
\hline & 1 & 2 & 3 & 5 & 8 & 13 & 21 & 34 & 55 & \\
\hline Total de SP & 4 & 0 & 3 & 5 & 16 & 91 & 21 & 68 & 0 & 213 \\
\hline Total de Casos de Uso & 4 & 0 & 1 & 1 & 2 & 7 & 1 & 2 & 0 & 23 \\
\hline A\&P & 10,00 & 0,00 & 2,50 & 4,50 & 12,00 & 42,00 & 8,00 & 48,00 & 0,00 & 127 \\
\hline Revisao de A\&P & 0,00 & 0,00 & 1,00 & 1,00 & 4,00 & 14,00 & 2,00 & 6,00 & 0,00 & 28 \\
\hline Codificação & 8,00 & 0,00 & 5,00 & 9,00 & 24,00 & 168,00 & 36,00 & \begin{tabular}{|l|l|}
104,00 \\
\end{tabular} & 0,00 & 354 \\
\hline Revisao de Codificação & 6,00 & 0,00 & 1,50 & 1,50 & 3,00 & 21,00 & 3,00 & 9,00 & 0,00 & 45 \\
\hline Especificação de testes & 10,00 & 0,00 & 4,00 & 6,00 & 12,00 & 70,00 & 13,00 & 26,00 & 0,00 & 141 \\
\hline Execução de Testes & 12,00 & 0,00 & 3,00 & 6,00 & 12,00 & 56,00 & 10,00 & 20,00 & 0,00 & 119 \\
\hline Total Geral & 46,00 & 0,00 & 17,00 & 28,00 & 67,00 & 371,00 & 72,00 & 213,00 & 0,00 & 814,00 \\
\hline
\end{tabular}

Figura 5: Planilha de estimativas de esforço dos casos de uso a partir de sua complexidade

Durante a fase Exploração, toda atualização do Backlog da Sprint com horas realizadas e restantes para se completar as tarefas do backlog era realizada na ferramenta JIRA. Tal ferramenta dispunha de consultas possibilitando uma configuração de dashboard para o time do projeto que facilitava reporte de horas, visualização dos impedimentos e pendências do projeto. O Gráfico de Consumo da Sprint também era acompanhado no JIRA. Os impedimentos registrados no JIRA eram tratados e resolvidos diariamente pelo Gerente de Projeto com a colaboração do time, que eram envolvidos por estratégias de motivação. Além das reuniões diárias, eram realizadas reuniões semanais formais de acompanhamento do projeto com o Gerente do Projeto e todo o time e reuniões quinzenais com a Gerência Sênior e cliente. O monitoramento dos riscos era realizado ao longo da execução da sprint sendo os riscos tratados nas reuniões diárias e semanais do projeto. Como todo o trabalho no projeto era realizado em sprints, o monitoramento do escopo e dos objetivos da sprint era realizado constantemente, avaliando-se o que seria possível entregar ao final da sprint, cumprindo-se assim os compromissos assumidos. 
Ao concluir a sprint, na fase Adaptação, era realizada uma análise do escopo planejado contra o realizado. A seguir, nas reuniões de revisão da sprint apresentavamse os resultados alcançados, incluindo escopo planejado x realizado, métricas do projeto e o sistema com as funcionalidades desenvolvidas na sprint. Por fim, realizavam-se a reunião de retrospectiva da sprint e a reunião com a Gerência Sênior para discutir indicadores do projeto, riscos e principais problemas. $\mathrm{Na}$ fase Encerramento foi realizada a celebração final do projeto em reconhecimento ao trabalho realizado com a liberação do time e da infra-estrutura do projeto.

Alguns desafios foram encontrados na aplicação do Scrummi como retratados em [Marçal 2009b]. O primeiro grande desafio do projeto piloto estava relacionado com a mudança cultural e comportamental no estilo de gerenciamento do projeto baseado na liderança e colaboração. Este novo estilo de gestão favoreceu a participação do time no planejamento e a construção de equipes auto-organizadas e auto-disciplinadas que compartilham a responsabilidade na execução do projeto. Além da liderança colaborativa, outro aspecto de mudança cultural importante no gerenciamento do projeto estava relacionado aos níveis de planejamento do projeto. Deixou-se de lado o planejamento detalhado realizado completamente no início do projeto e passou-se a adotar um planejamento incremental e iterativo, em que o detalhamento só é feito no início de cada sprint. Isto permitiu abraçar as mudanças ocorridas ao longo do projeto de forma natural e tranqüila, favorecendo a entrega de funcionalidades que atendiam aos requisitos do cliente e agregando valor ao seu negócio.

Outro grande desafio apostava na hipótese de melhoria de produtividade do projeto por meio da introdução de práticas ágeis dentro de um contexto de maturidade. A melhoria de produtividade (horas/Use Case Point) foi identificada logo no início do projeto após a execução das três primeiras sprints. Os resultados alcançados e medidos nas sprints seguintes confirmaram a alta produtividade do projeto o qual alcançou resultados cerca de 4 vezes melhor que a produtividade organizacional inicialmente estabelecida para o projeto.

Ao longo do projeto piloto também foi possível identificar vários resultados e lições aprendidas relacionadas com o uso de um processo de gestão ágil em um ambiente de maturidade como no caso do projeto piloto. Entre os principais resultados encontrados na aplicação do Scrummi pode-se citar: i) maior clareza e visibilidade do planejamento realizado a cada sprint pelo próprio time com a participação efetiva do cliente; ii) maior integração do time do projeto, sendo observado constante empenho de todos para fazer dar certo; iii) uso de estimativas rápidas em Story Points proporcionando maior agilidade no processo de planejamento; iv) implantação de uma cultura participativa no planejamento e gestão do projeto impondo credibilidade, transparência e comprometimento sobre o que se faz; v) autogerenciamento do time com amadurecimento gradativo; vi) avaliações e adaptações constantes do processo ao longo do projeto gerando aumento de produtividade a cada sprint.

Dentre as principais lições aprendidas no estudo destacam-se: i) a entrega constante de software funcionando é muito importante para a credibilidade do cliente com relação ao projeto realizado; ii) o autogerenciamento do time depende muito da sua maturidade (à medida que o time vai amadurecendo é possível deixá-lo mais à vontade para decidir sozinho quem faz o quê e quando); iii) é muito importante definir ou ter um processo de engenharia ágil, adequado às necessidades da organização e do projeto; iv) 
a colaboração e o comprometimento do cliente são fundamentais para o sucesso de um projeto que aplica práticas de gerenciamento ágil e participativo; v) o modelo de contratação dos projetos, baseado em um banco de horas é muito adequado ao contexto do gerenciamento ágil.

\section{Conclusões e Trabalhos Futuros}

O Scrummi conseguiu resolver todas as lacunas existentes e que impactam diretamente no planejamento e monitoramento do projeto representado pelas áreas de processo PP e PMC. Também resolveu todas as lacunas relacionadas com o gerenciamento de riscos e que afetam diretamente as práticas de RSKM, já que atividades específicas apoiadas por guias e templates foram definidas no processo visando identificar e analisar os riscos do projeto, bem como definir e acompanhar suas ações de mitigação e contingência. $O$ Scrummi deve ser complementado com processos organizacionais das empresas para se tornar $100 \%$ aderente à área de processo IPM.

A aplicação do Scrummi permitiu comprovar a possibilidade de adoção de práticas ágeis dentro de um contexto de maturidade contribuindo para a melhoria dos processos organizacionais e aumento de produtividade. $\mathrm{O}$ projeto piloto apresentado tornou-se uma referência na empresa com relação ao novo estilo de gerenciamento, o qual promove o desenvolvimento e comprometimento do time do projeto com alta motivação. Como principais contribuições do trabalho realizado durante esta pesquisa pode-se destacar: investigação da aderência do Scrum ao CMMI identificando os pontos fortes e problemas existentes; e definição e aplicação do Scrummi, um processo de gestão ágil simples e completo baseado no Scrum que é aderente às práticas de gerenciamento de projetos do CMMI. Com base nos resultados alcançados, considera-se que o Scrummi é útil para organizações que pretendem realizar a gestão ágil dos seus projetos sendo ao mesmo tempo compatível com práticas do CMMI.

Dentre os trabalhos futuros vislumbram-se: i) lançamento de uma nova versão do Scrummi, introduzindo melhorias já identificadas na execução do projeto piloto e que não foram ainda implementadas; ii) aplicação do Scrummi em outras organizações de forma que se possa identificar outras oportunidades de melhoria do processo; iii) expansão do Scrummi combinando-o com outras práticas do CMMI do nível 2 relacionadas com a definição e gestão de requisitos e métodos ágeis e; iv) estudo e análise de ferramentas que possam auxiliar na execução das atividades do Scrummi.

\section{Referências}

Alleman, G. (2004), Blending Agile Development Methods with CMMI. Cutter IT Journal, Vol 17, No 6, p. 5 -15.

Anderson, D. (2005), Stretching Agile to fit CMMI Level 3. Agile Conference, Denver. Beck, K. (1999) Extreme Programming Explained: Embrace Change. Addison-Wesley. Beck, K. et al. (2001), Manifesto for Agile Software Development.

Boehm, B.; Demarco, T. (2002), The Agile Methods Fray. IEEE Computer Science, p. 90-91.

Boehm, B; Turner, R. (2004), Balancing agility and discipline: a guide for theperplexed. Boston: Addison Wesley.

Boehm, B. (2006) A View of 20th and 21st Century Software Engineering. ICSE.

Chrissis, B. et al. (2007), CMMI Second Edition Guidelines for Process Integration and Product Improvement. Version 1.2. Addison-Wesley. 
Dalton, J. (2006), Agile CMMI: Process Innovation at the Speed of Life, SEPG 2006.

Davis, C. et al. (2007), An Agile Approach to Achieving CMMI.

Dutton, J. (2006), Agile / Lean Development and CMMI. SEPG 2006.

Glazer, H. et al., (2008) CMMI ${ }^{\circledR}$ or Agile: Why Not Embrace Both! Technical Note CMU/SEI-2008-TN-003, SEI, 2008.

Highsmith, J. (2004), Agile Project Management - Creating Innovative Products. Addison - Wesley.

Leal, L. (2006) Uma abordagem ágil ao gerenciamento de projetos de software baseada no PMBOK Guide. Orientador: Hermano Perreli. Dissertação de Mestrado. UFPE.

Marçal, A. S. et al. (2007a), Mapping CMMI Project Management Process Areas to SCRUM Practices. 31st Annual SEW, Loyola College, Baltimore, MD, USA.

Marçal, A. S. et al. (2007b), Estendendo o SCRUM segundo as Áreas de Processo de Gerenciamento de Projetos do CMMI. CLEI 2007, San Jose, Costa Rica.

Marçal, A. S. et al. (2008a) Blending Scrum practices and CMMI project management process areas. Innovations in Systems and Software Engineering Journal, Volume 4, Number 1 / April, Springer London.

Marçal, A. S. et al. (2008b), Uma Análise sobre o Interesse de Organizações na Melhoria de Processos de Gestão baseada em Práticas do Scrum e CMMI. CLEI 2008, Santa Fé, Argentina.

Marçal, A. S. (2008c) Scrummi: um processo ágil de gerência de projetos aderente ao CMMI. Fifth Edition of SEPG LA 2008, Mar del Plata - Argentina.

Marçal, A. S. et al. (2009a) Integração de Story Points e Use Case Points em Projetos Baseados em SCRUM e CMMI. SBQS 2009, Ouro Preto - MG.

Marçal, A. S. (2009b) SCRUMMI: Um processo de gestão ágil baseado no SCRUM e aderente ao CMMI. Orientadora: Elizabeth Furtado. Dissertação de Mestrado. UNIFOR.

Orr, K. (2002), CMM versus agile development: Religious Wars and Software Development. Cutter Consortium. Executive Report. Vol.3 No 7.

Paulk, M. (2001), Extreme Programming from a CMM Perspective, IEEE Software, vol. 18 , no. 6 , p.19-26.

PMI - Project Management Institute (2004). A Guide to the Project Management Body of Knowledge, 3a. edição, EUA.

Poppendieck, M. (2006) Lean Software Development: An Agile Toolkit, Agile Software Development Series.

Schwaber, K. (2004) Agile Project Management With Scrum, Microsoft Press, Redmond, Washington, USA.

Sutherland R. et al. (2007) Scrum and CMMI Level 5: The Magic Potion for Code Warriors. The 12th annual European Systems and Software Engineering Process Group Conference EUROPEAN SEPG 2007, 11-14th June, Amsterdam.

Turner, R.; Jain, A. (2002), Agile Meets CMMI: Culture clash or common cause. XP/Agile Universe. p.153-165.

Vriens (2003) C. Certifying for CMM Level 2 and ISO9001 with XP@Scrum. In Proceedings of the Agile Development Conference (ADC'03), pages 120-124, Salt Lake City, Utah, USA, IEEE Computer Society.

Zannata, A. L. (2004) xScrum: uma proposta de extensão de um Método Ágil para a Gerência e Desenvolvimento de Requisitos visando adequação ao CMMI. Orientadora: Patrícia Vilain. Dissertação de Mestrado. UFSC. Florianópolis. 